\title{
Anatomical variations in the superficial veins of head and neck and their clinical significance
}

\author{
Mohd.Saleem Itoo ${ }^{1}$, Shameema Banoo ${ }^{2}$, Gh.Mohd.Bhat ${ }^{3}$, Bashir Ahmed Shah ${ }^{1}$ \\ Omer Bashir Itoo ${ }^{4}$, Fahmida Akhter ${ }^{4}$, Mohd.Arif Makdomi ${ }^{4}$ \\ ${ }^{I}$ Assistant Professor Department of Anatomy Government Medical College Srinagar, J\&K,India \\ ${ }^{2}$ Lecturer Department of Anatomy Government Medical College Srinagar, J\&K,India \\ ${ }^{3}$ Associate Professor Department of Anatomy Government Medical College Srinagar, J\&K,India \\ ${ }^{4}$ Assistant Surgeon Department of Anatomy Government Medical College Srinagar, J\&K,India \\ *Corresponding author E-mail: dr.saleem68@gmail.com
}

\begin{abstract}
A very sound knowledge of variations of head and neck veins is important for General practitioners, plastic surgeons, interventional radiologists, internists and maxillofacial surgeons. Anatomic variations have a definite embryological basis and manifest their clinical impact. The Present study was conducted in the Department of Anatomy Government Medical College Srinagar to observe the variations in the pattern of superficial veins of head and neck. The aim of this study was to make clinicians academically wiser and more competent while dealing with these veins during surgery and other interventional procedures. Bilateral neck dissection of a formalin preserved middle aged male cadaver was done for routine Cadaveric anatomy teaching and simultaneously observations regarding the pattern of superficial veins of face and neck were recorded. It was observed that anomalous superficial veins were present in one cadaver on left side. The anomalous pattern of superficial veins of neck though rare is of great academic and clinical significance in plastic surgery, Otorhinology, Maxillofacial Surgery, Critical care medicine, interventional radiology and General Medicine. These variations should be kept in mind during surgery and other interventional procedures in this region.
\end{abstract}

Keywords: Anomalous veins, Central venous pressure, Common facial vein, Internal jugular vein, Retromandibular vein.

\section{Introduction}

The veins of head and neck have a complex developmental pattern which predisposes them to variations in their formation and drainage. On either side of face the superficial temporal vein unites with the maxillary vein to form retro mandibular vein within the substance of the parotid gland. The retro mandibular vein divides into anterior and posterior divisions. The anterior division joins with facial vein to form common facial vein inferior to the angle of mandible which drains into internal jugular vein. The posterior division joins with the posterior auricular vein to form the external jugular vein which drains into subclavian vein [2], [3].

The knowledge on the varied drainage patterns of these veins is important as they are often used for cannulation, monitoring central venous pressure, giving intravenous fluids/drugs in comatose and burn patients, where other superficial veins are not accessible. The retromandibular vein is an important anatomical guide in Radiology where it is used to localize a tumour in the parotid gland in relation to the facial nerve and in surgery as a land mark to dissect the facial nerve and its branches. The external jugular vein is used as venous manometer, while both the external and internal jugular veins are used for intravenous catheterization to carry out diagnostic procedures or intra venous therapies. The knowledge of these variations is important for plastic and vascular surgeons as these veins may be used as patches during carotid endarterectomy and for oral reconstruction surgeries, where facial vein is used for microvascular anastomosis. The external jugular vein is used for cannulation to conduct diagnostic procedures or intravenous therapies. The external jugular vein is easy to visualise than internal jugular vein and may give a reliable estimate of Central venous pressure (CVP). It is used to maintain central venous line and monitor central venous pressure (CVP) in comatose patients. Permanent catheterization for hemodialysis via it is a simple procedure without complications. The variations are also important for interventional radiologists who perform Transjugular procedures like port implantations and Transjugular intra hepatic Porto systemic shunts or selective venous sampling in patients with hyperparathyroidism of unknown origin [4]. 
The Present study was conducted in the Department of Anatomy Government Medical College Srinagar to study the variations in the pattern of superficial veins of head and neck. The aim of this study was to make clinicians extra know legible and more competent while dealing with these veins during surgery and other interventional procedures.

\section{Materials and methods}

During routine undergraduate dissection in the department of Anatomy Government Medical College Srinagar, variations in the superficial veins of head and neck were observed in a middle aged male cadaver on left side by dissecting it for routine Anatomic teaching purposes and recording observations. Bilateral neck dissection was done carefully and strictly following instructions given in the Cunningham's manual of practical Anatomy and observed for variations in the pattern of superficial veins of head and neck. After dissection of these veins cotton packs were kept posterior to them and these veins were stained with Indian ink to make them more prominent. Appropriate photographs were taken and labelled as shown in figure, [1].

\section{Case report}

During routine dissection variations in the pattern of neck veins were found in a middle aged Indian male cadaver on the left side. In this case facial vein received some tributaries from submandibular region (sub mental vein) to form a common anomalous venous trunk which joined with undivided retro mandibular vein and formed anomalous external jugular vein which opened into the subclavian vein whereas posterior auricular vein continued as a tributary of this vein

\section{Discussion}

There are available reports in the literature related to the variable course and pattern of veins of head and neck. The veins of head and neck have a complex developmental pattern which predisposes them to variations in their formation and drainage. The venous drainage of head and neck gets established after formation of skull. The first vessel that can be identified is ventral pharyngeal vein which drains mandibular and hyoid arches to the cardinal part of precardinal vein-the future internal jugular vein. The ventral pharyngeal vein receives tributaries from the face and the tongue and it becomes linguofacial vein. It anastomoses with the primitive maxillary vein to form the facial vein which receives a tributary from temporal region-the retromandibular vein and it drains into internal jugular vein through linguofacial vein. The external jugular vein arises in the neck region from a tributary of cephalic vein [1].The variations in the size and pattern of veins are quite common [2]. Cases have been reported in literature where facial vein terminates into external jugular vein [5].

There are also available reports in literature that retromandibular vein unites with the facial vein at a higher level in the parotid gland [6], the facial vein drained into the superficial temporal vein with an undivided retromandibular vein, the right facial vein drained into the superficial temporal vein about $5 \mathrm{~mm}$ above an undivided retromandibular vein [7] and the common trunk of the retromandibular ( RMV) joined with the anterior facial vein to form common facial vein with the absence of external jugular vein bilaterally. These anomalous venous patterns are due to the regression and /or the retension of venous anastomotic channels [8]. The superficial temporal vein and the retromandibular vein have been used as guides to expose the facial nerve branches in the parotid gland in superficial parotidectomy and also in cases of open reductions of the mandibular condyle fractures [9]. These veins are usually used as grafts during carotid endarterectomy and surgical microvascular anastomosis especially in oral reconstructive procedures [10]. Shahnaz Choudhary etal (2010) [11] reported a case where facial vein joined undivided retromandibular vein to form external jugular vein which received posterior auricular vein as tributary. She reports this pattern of veins bilaterally. The observations made in our present study were identical to those made by Shahnaz Choudhary etal except it was present only on left side. The variations/malformation in the pattern of superficial veins of head and neck being so common that they should be kept in mind in pre-operative period as this helps the surgeons in planning the operative procedures. 


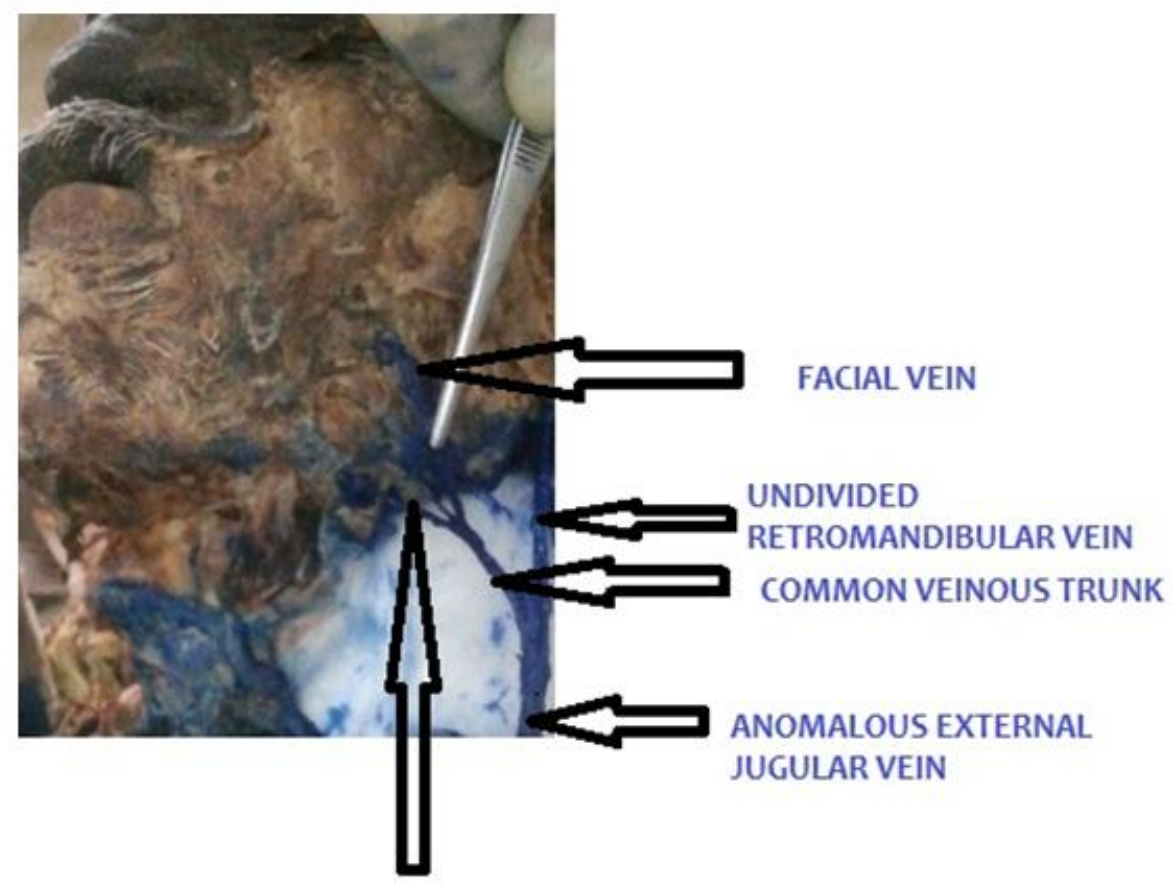

\section{SUB MENTAL VEIN JOINS FACIAL VEIN TO \\ FORM COMMON TRUNK}

Fig. 1: Showing Left sided facial vein joins with sub mental vein to form an anomalous venous trunk which in turn joins with undivided retro mandibular vein to form anomalous external jugular vein.

\section{Conclusion}

Anatomical variations of superficial veins of head and neck though rare, are of great academic importance. These variations have definite embryological basis and Clinical implications. They deserve due recognition and respect in academics and Clinical practice at both primary as well as tertiary levels of health care.

\section{References}

[1] Standring S.Gray's Anatomy-The anatomical basis of clinical practice, 39th edition Edinburg Elsevier Churchill Livingstone;2006,793794,225-226.

[2] Hollinshed W H.Anatomy for surgeons, The head and Neck 3rd edition.new yark ;harper and Row publishers;1968;530.

[3] Standring S. Gray's Anatomy. 39th Ed. Elsevier Churchil Livingstone. 2005; 1456-58.

[4] Sugg SL,Fraker DL,Alexander R,etal. Prospective evalvation of selective veinous sampling for para thyroid hormone concentration in patients undergoing reoperations for primary hyperparathyroidism surgery 1993; 114:1004-1010.

[5] Peuker ET,Fischer G,Filler TJ,The facial vein terminating in the superficial temporal vein,a case report.J Anat2001;198:509-10.

[6] Kopuz C ,llgi S,Yavuz S, Onderoglu S,Morphology of the retromandibular vein in relation to facial nerve in the parotid gland.Acta Anatomica (Basel).1995a,152(1),66:68

[7] Yadav S,Ghosh SK,Anad SC,Variations of superficial veins of the head and neck.j.Anat soc of India.2000;49:61-62.

[8] Bertha A,Rabi S.The anatomical variations in the termination of common facial vein.Journal of clinical and diagnostic research,2011 Feb;5(1);24-2.

[9] KawakamiS,Tsukada S,Taniguchi W.The superficial temporal and the retromandibulr veins as guides for exposing the facial nerve branches.Annals of plastic surgery.1994;32(2):295-99.

[10] Sabharwal P,Mukerjee D.the anomalous common facial vein or an external jujgular vein patch for carotid end arterectomy Cardiovascular surgery 1998;6:594-97.

[11] Shahnaz Choughary etal,Undivided retromandibular vein continuing as external jugular vein with facial vein draining into it, JK SCIENCE,Vol 12,NO. 4, OCT-DEC 2010 